\title{
Urban Air Mobility-A 6G Use Case?
}

\author{
Shuja Ansari *, Ahmad Taha, Kia Dashtipour, Yusuf Sambo, Qammer H. Abbasi and \\ Muhammad Ali Imran
}

James Watt School of Engineering, University of Glasgow, Glasgow, United Kingdom

The increasing popularity of Unmanned Aerial Vehicles (UAV) has resulted in exponential growth of the market owing to numerous applications that have been facilitated by advances in battery technology and wireless communications. Given the successes of UAVs thus far, researchers are already gearing towards aerial transport systems that consist of dense deployment of both UAVs and Personal Aerial Vehicles (PAVs) with human passengers. Although the fifth-generation mobile network (5G) key performance indicators have been optimised to support drone use cases for both high data rates and low latency applications, future aerial transport systems will require stricter network key performance indicators to support the expected massive deployment of aerial vehicles taking into

OPEN ACCESS

Edited by:

Xuan Liu,

Yangzhou University, China

Reviewed by:

Amin Shahraki, Fraunhofer Institute for

Communication, Germany

Muhammad Zakarya,

Abdul Wali Khan University Mardan,

Pakistan

Ons Aouedi,

Laboratoire des Sciences du Numérique à Nantes (LS2N), France

*Correspondence:

Shuja Ansari

Shuja.Ansari@glasgow.ac.uk

Specialty section:

This article was submitted to

Wireless Communications,

a section of the journal

Frontiers in Communications and

Networks

Received: 23 June 2021

Accepted: 06 August 2021

Published: 27 August 2021

Citation:

Ansari S, Taha A, Dashtipour K, Sambo $Y$, Abbasi $Q H$ and Imran $M A$ (2021) Urban Air Mobility-A 6G Use

Case?.

Front. Comms. Net 2:729767.

doi: $10.3389 /$ frcmn.2021.729767 account network capacity and distance between the base station and the aerial vehicles, among others. In this article, we present our perspective, vision, architecture, requirements and key performance indicators for future aerial wireless networks supported by $6 \mathrm{G}$ for Urban Air Mobility (UAM). Furthermore, we review key enabling technologies and discuss future challenges for incorporating aerial wireless networks in 6G.

Keywords: urban air mobility, 6G, wireless communication, autonomous flying, KPIs, UAV

\section{INTRODUCTION}

Radical innovations in technology and exponential increase in demand have been the key enablers for the evolution of mobile networks from the first-generation Total Access Communication Systems (TACS) to the fifth-generation communication systems (5G) which are now being deployed commercially. The Cisco VNI predicts that by 2022, 4G Long Term Evolution (LTE) traffic will constitute $71 \%$ of the total mobile connections, with $5 \mathrm{G}$ accounting for about $12 \%{ }^{1}$. One of the greatest developments in mobile communications was the transition from analogue (1G) to digital (2G) mobile telephony with the release of Global System for Mobile communications (GSM), giving a taste of data with General Packet Radio Service (GPRS) offering enhanced data rates ranging between 0.4-1 Mbps. Then the significant move towards spread spectrum disrupted 2G leading to what is now known as Universal Mobile Telecommunications Service (UMTS) or 3G, paving the way for mobile broadband with data rates of up to $42 \mathrm{Mbps}$. $4 \mathrm{G}$ was necessitated by the need for both spectral and energy efficiency, proposing scalable bandwidths and data rates of up to $150 \mathrm{Mbps}$. Innovation in waveform numerology and introduction of several other network functionalities took mobile communications from serving merely mobile phone users to serving everything that connects to the internet. 5G became truly application and user-centric. Interestingly, the switch between consecutive generations from $2 \mathrm{G}$ till $5 \mathrm{G}$, lasted roughly 10 years. With $5 \mathrm{G}$ now being commercially deployed, we are now starting to think about shaping our strategy for 6G Dang et al. (2020).

\footnotetext{
${ }^{1}$ https://newsroom.cisco.com/press-release-content?type=webcontent\&articleId=1955935
} 
Over the past year, a number of researchers have put forward their perspective and vision of the future $6 \mathrm{G}$ network. One of the most promising area that most authors have targeted for $6 \mathrm{G}$ is the NonTerrestrial Network (NTN) Giordani and Zorzi (2021); Kota and Giambene (2021). More recently, researcher at Ericsson published their perspective on the use of $6 \mathrm{G}$ for UAVs and beyond Mozaffari et al. (2021). All these works present an overview of their vision expanding on how the new and upcoming technologies will form part of the $6 \mathrm{G}$ network in supporting UAVs and NTN. Bekkouche et al. (2019) proposed a framework for the management and orchestration of UAVs' services in a Multi-access Edge Computing (MEC) Network Function Virtualization (NFV) environment by combining the functionalities provided by the management and orchestration framework with the functionalities of a UAV Traffic Management (UTM) system. Shrestha et al. (2021) also presented their perspective on the use of $6 \mathrm{G}$ for UAV traffic management. Their work focuses mostly on the UAV operation and its requirements whilst in operation, lacking some technical insights into mobile network technology and the requirements at the Key Performance Indicator (KPI) level.

While the industry remains busy deploying $5 \mathrm{G}$ over the terrestrial domain and the academia presents their perspective on the future mobile networks, we focus our research on utilizing $6 \mathrm{G}$ to enable the next generation of airborne transport systems. The main contributions of this paper are as follows:

1. A novel use case of Urban Air Mobility (UAM) for the 6th generation of mobile networks.

2. A vision and architecture for $6 \mathrm{G}$ Airborne Wireless Network (AWN) for UAM.

3. Key Performance Indicators (KPIs) of future 6G Networks enabling Astute Mobility.

4. The challenges, threats and future opportunities in the area of $6 \mathrm{G}$ and UAM.

The rest of the paper is organized as follows: the remaining of Section 1 talks about the evolution of smart mobility in mobile networks leading to the proposition of using mobile networks for Airborne Vehicles (AVs). Section 2 puts forward our vision and architecture for 6G AWN for UAM. In Section 3, we discuss the KPIs for $6 \mathrm{G} \mathrm{AWN}$ in line with UAM. Then in Section 4, we present some challenges, threats and opportunities for UAM and the next generation of mobile networks. Finally we conclude the paper in Section 5.

\subsection{Evolution of Smart Mobility in Mobile Networks}

Connected mobility dates back to 1946 when Bell Systems introduced the first ever car phone. This was a $36 \mathrm{~kg}$ phone installed in an automobile for the driver or passenger to make and receive phone calls. By 1980s the car phone was more popular than a regular mobile phone, however, with the 90's GSM technology and Bluetooth making mobile phones lighter and more accessible, they became a more popular option for in-car use Gregory et al. (2006). When the 3rd generation of mobile telephony offered Internet services, automobile manufacturers sought on installing $3 \mathrm{G}$ terminals as part of the vehicle multimedia, navigation and telematics services. Through this internet

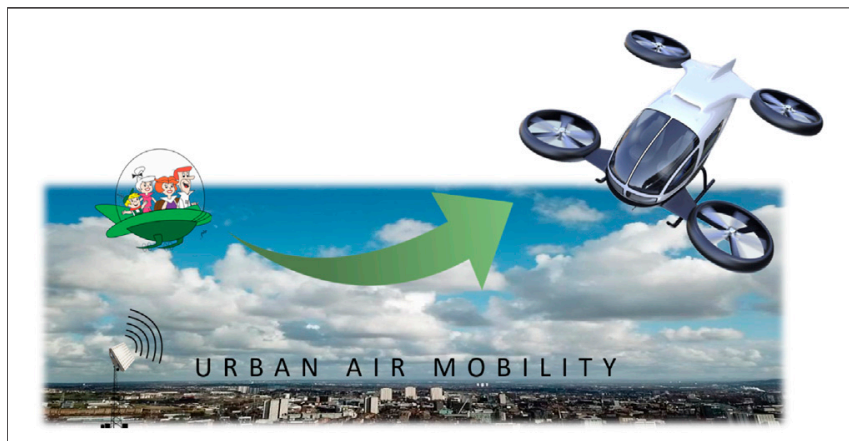

FIGURE 1 | A snapshot of Urban Air Mobility and Airborne Wireless Networks. Proposing 6G to enable Jetsons like Flying Cars in dense urban environments.

service, one could see live traffic in their area and download content from the internet. This was the first time when true online infotainment was offered to car users using mobile technology Kenis and Geerts, 2000.

By 2010s, with 4G LTE, similar in-car multimedia systems became more common, being up taken by more automobile manufacturers. With automobiles leaning towards electronics and software, some manufacturers used $4 \mathrm{G}$ to update car software over the air Coppola and Morisio (2016). Moreover, in 2018 at the CES exhibition in Las Vegas, Phantom Auto showcased controlling a vehicle remotely over commercial 4G network. However, their demonstration showed how higher data rates and lower latency is required for effective remote control and eventually autonomous driving Harris (2018). This intervention demanded the next generation of mobile networks to support these new mobility use cases. Therefore, one of the monumental use cases for $5 \mathrm{G}$ is Connected and Autonomous Vehicles (CAVs), where high data rates, low-latency and ubiquitous coverage allow vehicles to exchange vital data with the control centre and neighboring vehicles. Connectivity between the transport entities and infrastructure enables Intelligent Transport Systems (ITS) that in turn provide a range of applications including traffic management, road safety and infotainment services Nkoro and Vershinin (2014). CAVs and ITS consist of several strategic themes that address transport problems such as environmental sustainability, efficient travel, economy and connectivity.

For successful realization of CAVs it is crucial to have an efficient and robust communication system that considers different types of communications including vehicle to vehicle, vehicle to infrastructure, and vehicle to pedestrians to ensure a safe environment Postorino and Sarné (2020). 5G networks have proven to be instrumental in addressing these problems by providing connectivity to cars moving at speeds of up to $500 \mathrm{~km} / \mathrm{h}$ NGMN Alliance et al. (2015). Ground transport systems such as hyper-loop and bullet trains exceeding the $500 \mathrm{~km} / \mathrm{h}$ mark will require radio technologies that can provide connectivity to high speed User Equipment (UE). However, field trials for aerial UE suggest that there are still underlying problems pertaining RF characteristics 3GPP TR 36.777 (2017). In light with this evolution of mobile technology and mobility, this article discusses the role of 
cutting-edge next generation mobile communication technology for UAM, with particular focus on what $6 \mathrm{G}$ could offer, and any known or potential future challenges Li et al. (2020).

\subsection{Going Airborne}

With the disrupting innovations brought by $5 \mathrm{G}$ for vehicular communications, which in turn enabled CAVs, researchers started considering $6 \mathrm{G}$ as an enabler to AWNs Deebak and Al-Turjman (2020); Alsharif et al. (2020). AWNs are expected to unlock unprecedented applications for UAVs and subsequently Personal Aerial Vehicles (PAVs). UAVs refer to fixed wing or rotor blade drones and are of varying sizes, depending on the application. Whereas PAVs refer to aerial vehicles that can carry passengers. In other words, the success of PAVs can mean having flying cars in our environment, similar to The Jetsons as envisaged in Figure 1. Other examples of PAVs include the GL-10 developed by NASA, which was a two-seater electrically-powered Vertical Take-off and Landing (eVTOL) aircraft Uppal (2020).

UAM has already showed viability in various societal settings with UAVs and eVTOLs showing their applicability in a vast variety of fields. UAVs have the advantage of accessing places where human reach is difficult or costly, which prompted several industries to adopt them for numerous applications. This includes, remote inspection, maintenance, surveillance, cargo transport, film making, journalism, law enforcement, agriculture, construction, demining and for entertaining light shows Schelle and Stütz (2019). The list of these applications is quite comprehensive with additions being made every now and then. UAVs are also used as pop-up mobile networks for emergency disaster response to provide temporary mobile coverage Butterworth-Hayes (2020); Zhang et al. (2020). eVTOLs on the other hand have shown a lot of promise and increased confidence in air passenger transport through several successful tests and initiatives. This includes Uber's air-taxi pilot in Los Angeles, Dallas, and Dubai, and Hyundai's SA-1 air taxi revealed at the Consumer Electronics Show (CES) 2020 Uppal (2020).

Whilst there is evidence of progress towards UAM, there are still numerous gaps and challenges Ploetner et al. (2020). One challenge and a major requirement, addressed in this paper, is connectivity and coverage through AWNs. To enable a backhaul for such aerial services, much research efforts have been put into the development of AWN. These developments have investigated the challenges and opportunities for the aerial spectrum and its uses Giovanni Geraci et al. (2019). Since UAVs will act both as UEs and base stations (aerial NodeB?) as specified by 3GPP TR 36.777 (2017), it will bring upon the complexity of having dual mobile transceivers, putting forth energy consumption related challenges. Hence, even though there are several UAV applications, many research questions still loom over our heads.

Recent advances in electric batteries and drone technology supported by numerous development efforts by automobile and aircraft manufacturers make the development of PAVs highly likely in the near future. Just a decade ago, autonomous vehicles were considered improbable and now we have them being

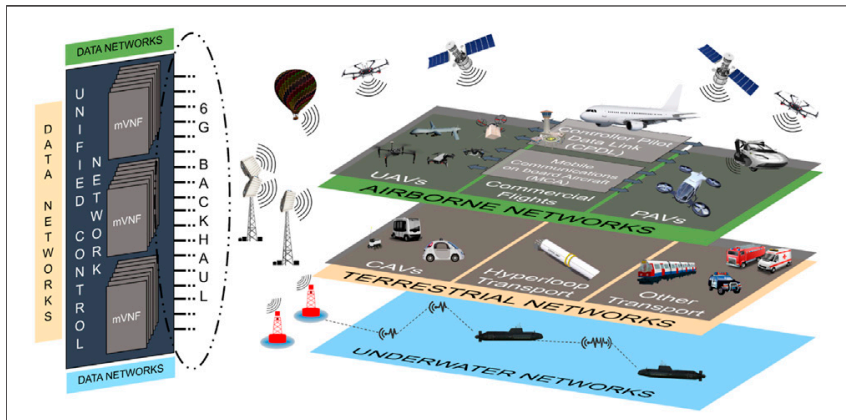

FIGURE 2 | Envisaged Hierarchical 6G Architecture for airborne, ground and underwater networks with a distributed data backhaul including massive Virtual Network Functions and a unified control network.

deployed in developed spaces. Vehicular communications have played a major role in the success of CAVs, where low latency and ultra reliable communications made numerous CAV applications possible.

Considering PAVs will be fully autonomous, one of the major advantage PAVs will have over CAVs is the coexistence with human driven vehicles. The most challenging aspect of autonomous vehicle deployment is their integration with normal traffic, which can be avoided for autonomous aerial mobility if we start the deployment of PAVs as fully autonomous from the very beginning of their development.

Considerable efforts are being put forward by automobile, aircraft and other manufacturers to enable urban air mobility. Japanese company NEC showcased their PAV last year and expects to have them deliver goods by 2023 while people to ride in them by 2030 NEC (2020). Similar to aircraft traffic management, these PAVs will require reliable positioning services along with robust communications with control centres that will ensure safety and efficiency along their routes. Although aeronautical telecommunication networks exist, they require an upgrade to increase resilience, security and reliability since in the case of on-board autonomous pilot system failure, remote control of the PAV will also require sub millisecond latency and a six nine's reliability She et al. (2019).

\section{Airborne Wireless Network (6G) for Urban Air Mobility: Vision and Architecture}

The 6G mobile network is expected to heavily utilize Artificial Intelligence (AI) to dynamically and intelligently configure and organise its resources according to the service type and environment it serves. We also envisage AI to be instrumental for airborne networks serving high speed ground and AVs since the fast changing topology will require the network to adapt to rapid channel and environment changes proactively. Dang et al. (2020).

Figure 2 shows our vision of a unified $6 \mathrm{G}$ control plane network being fed with information from contiguous data networks providing connectivity to ground, airborne and 
underwater networks. At the core side of the network, massive Virtual Network Functions (mVNFs) are expected to serve the large number of applications. A unified control network will enable coexistence of several network entities while data services can be provided by various service providers placed according to their application requirements through the presented $6 \mathrm{G}$ network. Low Earth Orbit (LEO) satellite constellations such as the Starlink by SpaceX, OneWeb, Project Kuiper by Amazon, etc will be an integral part of $6 \mathrm{G}$, providing connectivity to the aerial UEs and backhaul for radio access networks, particularly for ultra-dense and remote deployments. Furthermore, high altitude platforms such as high-altitude balloons including the Project Loon LLC by Alphabet could also be used to beam internet connectivity to remote areas. Using similar platforms and comprehensive AI-based RF planning in the 3-dimensional space, we can deploy mobile networks to provide connectivity to AVs. Base stations (gNBs) with antennas tilted upwards like the European aeronautical telecommunication network EUATN (2020), can also provide connectivity to aerial UEs. Such globally ubiquitous connectivity will further complement the existing terrestrial networks. Additionally, with massive satellite coverage of the globe, we envisage that underwater communications will also utilize the 6G backhaul connectivity for supporting the marine ultra massive Machine Type Communications (umMTC) Alsharif et al. (2020).

UAVs and PAVs are envisaged to act both as a UE and a base station. Since there will be substantial network intelligence involved in the $6 \mathrm{G}$ networks, the core network will be able to dynamically decide whether a NodeB will act as a ground NB (gNB) or an aerial-NodeB (aNB) depending on the environment, application and/or other required specifications. As a UE, the AV can enable its control and applications such as providing airspace management, clearance, safety, infotainment, etc. Whereas, as a gNBs or aNB, it can provide connectivity to either its occupants in the case of PAVs or neighbouring AVs/UEs in the case of UAVs. 3GPP considers these UAVs and PAVs as Aerial UE (AUE) where they have studied several radio planning strategies and have presented us with the several challenges for AUEs 3GPP (2017).

Another significant factor to be considered as an integral design of a network for UAM is security. As the mobile networks widen their scope, new applications and heightened network usage call for stricter security measures. Security breaches to critical applications could potentially result in a fatality. Substantial efforts have been put into $5 \mathrm{G}$ network design to address security. Emerging technologies such as quantum computing and Distributed Ledger Technology (DLT) can play a vital role in security of $6 \mathrm{G}$ networks. The use of Quantum Key Distribution (QKD) for increasing network security using UAVs and satellites to distribute the keys has found much attention from the research community Sasaki (2017). At the same time, DLT can address privacy issues for direct communications and also for the Internet of Things domain Dorri et al. (2017). Over the next decade, researchers and scientists will work on technologies for cybersecurity that as we envisage will make $6 \mathrm{G}$ the most secured mobile network.

\subsection{Airspace Division and Radio Network Planning}

The world's airspace is divided into three-dimensional segments which are further assigned to specific classes. International commercial airlines adhere to classification specified by the International Civil Aviation Organization (ICAO), however, countries might use only some of the defined classes and significantly alter the exact rules and requirements NATS (2020). 6G enabled airborne networks have to be able to support air traffic control for aircraft identification and subsequent traffic flow. We envision that certain airspace classes that are not fully controlled can be further revised and extended with addition of autonomous air traffic control aspect to play a vital role in enabling AWNs.

With additional emerging applications of UAVs and realisation of PAVs, these airspace classes will require subsequent radio planning. Future urban AVs will mostly operate in low-altitude airspace. UAVs are already limited to 400 feet above the ground in the United Kingdom, whereas, PAVs might be allowed to use slightly higher altitudes with limit going up to 2000 feet above ground Elias (2018). Traditional radar systems and radio communications are not viable for tracking and controlling low-flying aircraft in dense urban scenarios. The National Aeronautics and Space Administration (NASA) has been tasked with the research and development of airspace management for UAVs while Amazon and Google are reportedly working towards the development of a private airspace system for controlling low-altitude airspace. This calls for a system that establishes dynamic airway corridors for UAVs and PAVs to utilize. Space RF planning combined with AI and massive number of airborne antennas could enable the formation of on-demand dynamic airway corridors, which will be an important stepping stone towards UAM.

\subsection{Envisaged 6G Technologies for UAM}

Over the past three decades, three main general strategies have been employed to achieve high capacity, i.e., densification of infrastructure, larger bandwidths and large number of antennas. Will we adopt the same strategy for 6G? Since the challenges with airborne networks are of different nature, we believe these strategies alone cannot address the unique challenges. In the following subsections, we outline the most important technologies and strategies that we expect $6 \mathrm{G}$ to have that in turn will enable UAMs.

\subsubsection{Uncharted Frequency Bands}

The unique characteristics of millimetre wave and Terahertz $(\mathrm{THz})$ band set them aside from the traditional mobile telephony frequency bands. Millimeter wave found its place in $5 \mathrm{G}$ providing high bandwidths and substantial data rates with a coverage trade-off. Similarly, the $\mathrm{THz}$ band is envisaged as an important wireless technology that can satisfy the real-time traffic demands by diminishing the spectrum scarcity and capacity 
limitations. Several recent advancements in $\mathrm{THz}$ have enabled their practical use for communications. Some research directions for the development of $\mathrm{THz}$ transceiver front-ends that operate at ultra-high spectral efficiency with bandwidths of $50 \mathrm{GHz}$ employing higher-modulation schemes are presented in Boulogeorgos et al. (2018). There are still many challenges in realizing these frequency bands for AWNs, however, we envisage massive Multiple-Input and Multiple-Output (MIMO) and relaybased communications can make $\mathrm{THz}$ band practical. Furthermore, since the $\mathrm{THz}$ band cannot reach farther distances, it can be used for airborne Vehicle-to-Vehicle (V2V) applications for vehicles within range. With mostly Line-of-Sight (LoS) operations for AWN, the spectrum in the mmWave region and $\mathrm{THz}$ band can open up important research and development opportunities, in the field, in order to make them viable for airborne networks.

\subsubsection{Intelligent Reflecting Surfaces}

Intelligent Reflecting Surfaces (IRS) are antenna arrays that move beamforming functionalities from the radio transceivers to the propagation environment Arun and Balakrishnan (2019). IRS is an inexpensive RF switch that either lets the signal through or reflects it depending on the transmission requirement. These surfaces not only increase coverage but can also preserve privacy. Some of the current challenges with IRS include development of practical protocols, channel sensing, HetNet operations, etc Gong et al. (2019). In terms of UAM, IRS mounted UAVs and buildings can act as cost effective relays, further enhancing AWN coverage and promoting Beyond Visual Line-of-Sight (BVLoS) UAV operations. We envisage these will be used at places where cost effective coverage and throughput solutions are required Abadal et al. (2020). The challenge with IRS for UAM will be to determine where and when they can be used effectively. Research will need to focus on these challenges to make them viable for 6G AWNs.

\subsubsection{Massive Intelligence}

As AI becomes more and more viable, it will find its place in all the aspects of mobile networks. From radio planning to network orchestration and from user experience to operator policies, all these operations will find some feature of AI. Bonawitz et al. (2019) proposed federated learning where a model can be trained on a large corpus of decentralized data using distributed machine learning. Similar principles can be adopted for federated analytics, where mobile core network can monitor aggregate device statistics without logging raw device data to the cloud. These principles make a good case for AI to be an integral part of 6G airborne networks to enhance and maintain the network performance autonomously. More specifically, the change in aerial environment and topologies will require the network to adjust accordingly and we envisage AI will play a major role in network orchestration and radio optimisation for AWN.

\subsubsection{Low Earth Orbit Satellite Backhaul}

Increased research and development efforts are being observed on the use of LEO satellites for backhauling traffic for the next generation mobile networks Li et al. (2020). Satellites have better

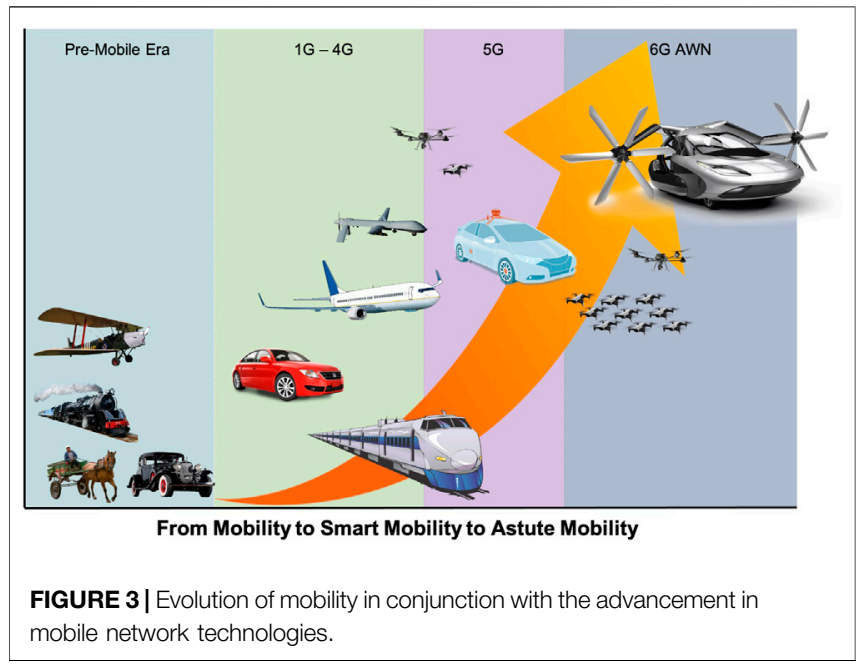

coverage when compared with terrestrial networks due to how far satellites are from earth, which comes with the resultant challenge of much higher latency. With recent advances in radio networks, for instance, the use of Multi-access Edge Computing (MEC), higher frequency bands and cost practicalities of high throughput satellites, their use in AWNs, offering low latency aerial mobile communications can unlock several AV applications. Companies like Amazon and SpaceX have already started investing in high throughout LEO satellite constellations, namely Project Kuiper and Starlink, to provide global internet coverage. These constellations will play an instrumental role in enabling $6 \mathrm{G}$ mobile telephony.

\subsubsection{Quantum Computing}

Cybersecurity is a major challenge and with increasing control and data intensive real-time applications, data privacy, and security requirements also increase Maxa et al. (2019). Security will be the most critical in 6G based AWNs, especially in the case of serving safety and control related applications to AVs where a security or privacy breach possibly leading to hijack of controls can have grave consequences. QKD for communications relies on the principles of quantum mechanics where two communicating users can detect the presence of any third party trying to gain access to the key. Main challenge of QKD is that it requires an already existing authenticated channel of communications to be able to exchange the keys. Some studies have suggested the use of satellites and UAVs Quintana et al. (2019) for key generation and distribution. There is still a lot of research and development required to realize $\mathrm{QKD}$ for $6 \mathrm{G}$ communications, however, opportunity lies for $\mathrm{QKD}$ in the next generation of mobile networks.

\section{6G AWN KPIS-ENABLING ASTUTE MOBILITY}

In order to achieve true application driven networking for 5G, various use cases and their requirements were first identified, 
followed by the development of technology. In terms of mobility, connected cars make our roads safer, travels shorter and environment healthier, while addressing the socio-economic benefits of transport. Intelligent mobility addresses end-to-end movement of people and goods while seeking a balance between cost, comfort, speed and convenience against our needs. 6G enabled UAM services will share data behind the scenes to dynamically adjust based on demand and synchronise with other services using network intelligence. We expect $6 \mathrm{G}$ will play a leading role in the management of aerial transport, and over the next decade, use cases and applications that will bring more value to our mobility will be defined.

In the following, we discuss some mobile network KPIs and the role they will play in evolving our mobility and subsequently AWNs (Figure 3).

Accessibility: This is a significant network and user side performance indicator that evolves as the network evolves. For $6 \mathrm{G}$ networks, where multiple network functionalities at the core will be shared, and user applications will rely on massive network function virtualisation, performance measures that specify whether the user of a service has received successful registration for a specific service or not will be invaluable. The evolution of this KPI will depend on the performance and capabilities of network technologies as they progress towards the next generation. In terms of aerial mobility, evolution and scaling up of current network technologies will be inevitable to accommodate the new set of users. Non-accessibility of the network can have serious consequences, as these aerial vehicles will rely on aerial networks to operate.

Integrity: This specifies the degree to which a service is provided without any unwarranted impairments. Emerging applications for $6 \mathrm{G}$, requiring higher data rates and lower latencies will put much stress on the integrity KPI. In addition to latency and throughput, data security and privacy will become a major part of integrity KPI for 6G. Data integrity is significantly important for applications involving human life on stake. Aerial mobility, including UAVs and PAVs, will make use of AWNs for navigation, taking off and landing manoeuvres. In such circumstances, unwanted delay or a cybersecurity attack can result in catastrophic consequences.

Utilization: This KPI focuses on the consumption of network resources as the application usage grows. This KPI is significant as it evaluates the utilisation of network resources and helps in determining whether any network scale up or scale down operations are required or not. Self-organising networks, as specified in 3GPP TR 22.829 (2019), take into account this KPI to organise virtual resources and configure network architecture accordingly. With a steep increment in network usage by airborne network entities, this KPI will be key for 6G to address the user/ application data consumption and give insights to operators/ management entities for better decision making.

Retainability: This KPI measures the preservation of Quality of Service (QoS) flow associated with each user application. QoS is vital for users to achieve the quality of experience and network providers to avoid customer churn. Radio and network resources can be scarce; hence monitoring of QoS is vital. For airborne network applications, the transmission requirements for safety and traffic management applications will be extremely strict. Hence, stringent QoS flows will be required to address emerging aerial applications. Nevertheless, this KPI will be vital in determining the success of $5 \mathrm{G}$ networks and subsequent $6 \mathrm{G}$ airborne networks.

Mobility: Until 5G, mobility involved successful handovers between gNBs and Next Generation Radio Access Networks (NG-RANs) while maintaining the end-to-end link to the network slice. With aNBs, the mobility for $6 \mathrm{G}$ will need to cater for handovers in the $3 \mathrm{D}$ airspace for instance between the ground and aerial base stations. The success of these handovers depends on many factors including radio network planning, network orchestration and availability of resources. Efficient mobility is important for fast-moving vehicles, and more significantly for AVs. 3GPP TR 36.777 specifies some enhancements in LTE for aerial vehicles where the mobility KPIs further include handover rate, handover failure rate, radio link failure rate, handoff times and ping pong rates. To enhance this KPI, AI will play its role in making handovers in airborne networks predictive and seamless.

Nevertheless, poor radio planning can cause substantial deterioration in all the above KPIs, leading to inadequate network performance and substandard user experience.

\section{CHALLENGES, THREATS AND OPPORTUNITIES}

There are many challenges on our way to $6 \mathrm{G}$ AWN. The enabling technologies for AWN will address these challenges and pave way for airborne networks and astute mobility. In this section, we discuss some of the main challenges and threats followed by a brief summary of some future research directions.

The United Kingdom Civil Aviation Authority (CAA) has developed an elaborate system to control the airspace. The aircraft generally follows the predefined routes called airways that are implemented to connect heavy traffic regions while ensuring adequate radar coverage for controlling traffic. Furthermore, the existing radio network that assists large aircraft instruments and pilots in all operations of a flight is a complex harness of electromagnetic spectrum. Considerable development and testing efforts are required in revising and planning radio networks for massive aerial mobility. The challenge will be to extend this airspace control system to include AWN entities and subsequent aerial vehicles in an official and elaborate manner to ensure safety, resilience and efficiency.

Cybersecurity is also one of the most important issues in the air traffic control system since many functionalities are heavily dependent on communication between the network agents. Potential mechanisms to protect airborne communications from cyber-attacks are required. GPS spoofing, false data injection and similar cyber-attacks are a major threat to the safety of aerial mobility. There are still many unanswered questions and concerns in terms of data and control integrity in addition to regulatory and compliance issues that will be major obstacles in the deployment and adoption of aerial mobility. Significant research is needed to address security and privacy concerns.

As evident from 3GPP studies and field trials, problems like interference and energy efficiency are also the most challenging 
aspects of aerial UEs. At the same time, operators and service providers will ponder upon RF planning in the $3 \mathrm{D}$ airspace. Proper harnessing of the electromagnetic spectrum will be important in planning the airborne networks. In light of the above challenges, we require techniques and mechanisms that address these concerns and evolve our mobile networks in the right direction.

We list below some of the future research directions and opportunities that we envisage will enable the 6th wave of mobile technologies for mobility use cases as presented in this article.

Network and Application Intelligence: AI can make our networks smarter and proactive to the environment, similarly, developing features like predictive handovers and AI-assisted channel sensing can address aerial network problems like interference and energy efficiency.

THz Transceivers: Development of transceivers and antenna arrays utilizing the $\mathrm{THz}$ band will be challenging. For applications like internet of nano things and connected micro-mobility, such bands will be instrumental. Beam-tracking of these waves specially in moist air environment will require investigation to foresee their practicality. At the same time, integrating beyond mmWave bands and IRS with 6G airborne networks is an open challenge that requires substantial research efforts.

6G Satellite: High-throughput satellites are ideal for providing unprecedented coverage and capacity, and given that UAM requires ubiquitous coverage, we shed some light on challenges of LEO satellite constellations for $6 \mathrm{G}$ mobile communications. Challenges include securing highly reliable, flexible and scalable $6 \mathrm{G}$ links at a viable cost while technical challenges include managing the frequent handovers between high velocity LEO satellites, seamless integration of robust and ultra-low latent inter-satellite routing algorithms. With all these challenges, there are several research opportunities in the field of LEO satellites for 6G and more particularly AWNs.

Intelligent Reflecting Surfaces: Given the need for ubiquitous coverage for UAM, programmable, software defined and passive IRS metasurfaces can be used as inter-satellite radio switches presenting numerous technical solutions to coverage and capacity problems. These smart surfaces mounted along or either in part of the solar panels can also play an enabling role in reducing energy consumption for satellite communication. Major implementation challenge include tracking high mobility satellites in order to maintain links in real-time with minimum power consumption.

Security and Privacy: Due to the nature of mobile communications, research on cybersecurity will always remain ongoing. Efforts are required to secure our mobile networks

\section{REFERENCES}

3 GPP TR 22.829 (2019). “3rd Generation Partnership Project; Technical Specification Group Services and System Aspects; Telecommunication Management," in Study on the Self-Organizing Networks (SON) for 5G Networks (Release 16) 0, 1-53.

3 GPP TR 36.777 (2017). "3rd Generation Partnership Project; Technical Specification Group Radio Access Network," in Study on Enhanced LTE Support for Aerial Vehicles (Release 15).

3 GPP (2017). TR 36.777 Field Trials Results on Mobility. specifically for applications that involve human life at risk. Vulnerability in communication links for AWNs can simply result in hijack of controls or data breaches, hence demanding much research efforts in addressing the security and privacy challenges.

\section{CONCLUSION}

In this paper, we have presented our vision of $6 \mathrm{G}$ AWNs enabling urban air mobility. A 6G architecture for airborne networks is presented and enabling technologies specific to aerial use cases are discussed in detail. We envisage airborne networks utilizing aerial vehicles as base stations (aNBs) to enable a multitude of applications for future astute mobility. We also examine the required evolution of KPIs for $6 \mathrm{G}$ airborne networks and UAM applications. Finally, we discussed the challenges and threats in pursuing AWNs and subsequent future research opportunities in the space of UAMs and 6G. Our work in this paper highlights the need of rigorous research efforts in bringing the vision to life, however, these insights and past developments of mobile networks and their respective applications make a solid case for the realisation of Astute Urban Air Mobility.

\section{DATA AVAILABILITY STATEMENT}

The original contributions presented in the study are included in the article/Supplementary Material, further inquiries can be directed to the corresponding author.

\section{AUTHOR CONTRIBUTIONS}

SA, AT, KD, YS, and QA contributed to conception and design of the study. SA wrote the first draft of the manuscript. AT, KD, YS, $\mathrm{QA}$, and MI wrote sections of the manuscript. All authors contributed to manuscript revision, read, and approved the submitted version.

\section{FUNDING}

This work is supported in parts by engineering and physical council (EPSRC) grant no EP/R511705/1.

Abadal, S., Cui, T., Low, T., and Georgiou, J. (2020). Programmable Metamaterials for Software-Defined Electromagnetic Control: Circuits, Systems, and Architectures. IEEE J. Emerging Selected Top. Circuits Syst. 10, 6-19. doi:10.1109/jetcas.2020.2976165

Alliance, B., El Hattachi, R., and Erfanian, J. (2015). NGMN 5G White Paper. Tech. rep.

Alsharif, M. H., Kelechi, A. H., Albreem, M. A., Chaudhry, S. A., Zia, M. S., and Kim, S. (2020). Sixth Generation (6G) Wireless Networks: Vision, Research Activities, Challenges and Potential Solutions. Symmetry 12, 676. doi:10.3390/sym12040676

Arun, V., and Balakrishnan, H. (2019). "RFocus: Beamforming Using Thousands of Passive Antennas," in Proceedings of the 17th Usenix Conference on 
Networked Systems Design and Implementation, Santa Clara, CA (Berkeley, CA: USENIX Association), 1047-1062.

Bekkouche, O., Bagaa, M., and Taleb, T. (2019). "Toward a Utm-Based Service Orchestration for Uavs in Mec-Nfv Environment," in 2019 IEEE Global Communications Conference (GLOBECOM), Waikoloa, HI, December 9-13, 2019. doi:10.1109/globecom38437.2019.9014200

Bonawitz, K., Eichner, H., Grieskamp, W., Huba, D., Ingerman, A., Ivanov, V., et al. (2019). Towards Federated Learning at Scale: System Design. Tech. rep.

Boulogeorgos, A.-A. A., Alexiou, A., Merkle, T., Schubert, C., Elschner, R., Katsiotis, A., et al. (2018). Terahertz Technologies to Deliver Optical Network Quality of Experience in Wireless Systems beyond 5G. IEEE Commun. Mag. 56, 144-151. doi:10.1109/MCOM.2018.1700890

Butterworth-Hayes, P. (2020). Drones Alongside 5G to Solve Poor Network Connection during Crowded Events - Urban Air Mobility News. https://www. urbanairmobilitynews.com/uncategorized/drones-alongside-5g-to-solve-poornetwork-connection-during-crowdedevents/.

Coppola, R., and Morisio, M. (2016). Connected Car: Technologies, Issues, Future Trends. ACM Comput. Surv. 49, 1-36. doi:10.1145/2971482

Dang, S., Amin, O., Shihada, B., and Alouini, M.-S. (2020). What Should 6G Be? Nat. Electron. 3, 20-29. doi:10.1038/s41928-019-0355-6

Deebak, B. D., and Al-Turjman, F. (2020). Drone of IoT in $6 G$ Wireless Communications: Technology, Challenges, and Future Aspects. Cham: Springer International Publishing, 153-165. doi:10.1007/978-3-030-38712-9_9

Dorri, A., Kanhere, S. S., Jurdak, R., and Gauravaram, P. (2017). "Blockchain for IoT Security and Privacy: The Case Study of a Smart home," in 2017 IEEE International Conference on Pervasive Computing and Communications Workshops, PerCom Workshops 2017 (Institute of Electrical and Electronics Engineers Inc.), 618-623. doi:10.1109/PERCOMW.2017.7917634

Elias, B. (2018). CRS INSIGHT Flying Cars and Drones Pose Policy Challenges for Managing and Regulating Low-Altitude Airspace Related Author. Tech. rep.

EUATN (2020). EUROPEAN AERONAUTICAL TELECOMMUNICATION NETWORK - EURATN Project - FP2 - CORDIS - European Commission. https://cordis.europa.eu/project/id/81011402.

Geraci, G., Garcia-Rodriguez, A., and Lin, X. (2019). Preparing the Ground for Drone Communications (IEEE ComSoc Technology News (CTN). https://www. comsoc.org/publications/ctn/preparingground-drone-communications.

Giordani, M., and Zorzi, M. (2021). Non-terrestrial Networks in the $6 \mathrm{~g}$ Era: Challenges and Opportunities. IEEE Netw. 35, 244-251. doi:10.1109/ mnet.011.2000493

Gong, S., Lu, X., Hoang, D. T., Niyato, D., Shu, L., Kim, D. I., et al. (2019). Towards Smart Radio Environment for Wireless Communications via Intelligent Reflecting Surfaces: A Comprehensive Survey. IEEE Commun. Surv. Tutorials 22 (4), 2283-2314. doi:10.1109/COMST.2020.3004197

Gregory, D., and Bates, R. J. (2006). Voice \& Data Communications Handbook. New York, NY, United States: McGraw Hill Professional.

Harris, M. (2018). Ces 2018: Phantom Auto Demonstrates First Remote-Controlled Car on Public Roads. IEEE Spectr. Technol. Eng. Sci. News. https://spectrum.ieee.org/ces2018-phantom-auto-demonstrates-firstremotecontrolled-car-on-public-roads.

Kenis, E., and Geerts, S. (2000). "Flanders: Case-Study and Demonstration of a Third Generation In-Car Telematics Infrastructure. Functional and Organisational Issues," in PROCEEDINGS OF THE 7TH WORLD CONGRESS ON INTELLIGENT SYSTEMS, Turin, Italy, November 6-9, 2000.

Kota, S., and Giambene, G. (2021). "6g Integrated Non-terrestrial Networks: Emerging Technologies and Challenges," in 2021 IEEE International Conference on Communications Workshops (ICC Workshops), Montreal, QC, July 9, 2021. doi:10.1109/iccworkshops50388.2021.9473743

Li, X., Feng, W., Chen, Y., Wang, C.-X., and Ge, N. (2020). Maritime Coverage Enhancement Using UAVs Coordinated with Hybrid Satellite-Terrestrial Networks. IEEE Trans. Commun. 68, 2355-2369. doi:10.1109/tcomm. 2020.2966715
Maxa, J. A., Blaize, R., and Longuy, S. (2019). "Security Challenges of Vehicle Recovery for Urban Air Mobility Contexts," in 2019 IEEE/AIAA 38th Digital Avionics Systems Conference (DASC), 1-9. doi:10.1109/DASC43569.2019.9081808

Mozaffari, M., Lin, X., and Hayes, S. (2021). Towards 6g with Connected Sky: Uavs and beyond. CoRR abs/2103.01143

NATS (2020). Introduction to Airspace - National Air Traffic Services. https://www. nats.aero/ae-home/introduction-to-airspace/.

NEC (2020). eVTOL Testbed - Electric VTOL News ${ }^{T M}$. https://evtol.news/necflying-car/.

Nkoro, A. B., and Vershinin, Y. A. (2014). "Current and Future Trends in Applications of Intelligent Transport Systems on Cars and Infrastructure," in 17th International IEEE Conference on Intelligent Transportation Systems (ITSC), 514-519. doi:10.1109/ITSC.2014.6957741

Ploetner, K. O., Al Haddad, C., Antoniou, C., Frank, F., Fu, M., Kabel, S., et al. (2020). Long-term Application Potential of Urban Air Mobility Complementing Public Transport: an Upper Bavaria Example. CEAS Aeronaut. J. 11, 991-1007. doi:10.1007/s13272-020-00468-5

Postorino, M. N., and Sarné, G. M. L. (2020). Reinventing Mobility Paradigms: Flying Car Scenarios and Challenges for Urban Mobility. Sustainability 12, 3581. doi:10.3390/SU12093581

Quintana, C., Sibson, P., Erry, G., Thueux, Y., Kingston, E., Ismail, T., et al. (2019). "Low Size, Weight and Power Quantum Key Distribution System for Small Form Unmanned Aerial Vehicles," in Free-Space Laser Communications XXXI, SPIE LASE, 2019 (San Francisco, CA: International Society for Optics and Photonics), 10910, 1091014. doi:10.1117/12.2507669

Sasaki, M. (2017). Quantum Networks: where Should We Be Heading? Quan. Sci. Technol. 2, 020501. doi:10.1088/2058-9565/aa6994

Schelle, A., and Stütz, P. (2019). "Visual Communication with UAV: Use Cases and Achievements," in Communications in Computer and Information Science (Salerno, Italy: Springer-Verlag), 1089, 120-128. doi:10.1007/978-3-030-29930-9_12

She, C., Liu, C., Quek, T. Q. S., Yang, C., and Li, Y. (2019). Ultra-Reliable and LowLatency Communications in Unmanned Aerial Vehicle Communication Systems. IEEE Trans. Commun. 67, 3768-3781. doi:10.1109/tcomm.2019.2896184

Shrestha, R., Bajracharya, R., and Kim, S. (2021). 6g Enabled Unmanned Aerial Vehicle Traffic Management: A Perspective. IEEE Access 9, 91119-91136. doi:10.1109/access.2021.3092039

Uppal, R. (2020). Urban Air Mobility, the Future of Urban Transportation Requires New Technologies - International Defense Security \& Technology Inc. https:// idstch.com/military/air/new-technology-requirements-forurban-air-mobilitythe-future-of-urban-transportation-requires/.

Zhang, H., Song, L., and Han, Z. (2020). UAV Assisted Cellular Communications. Cham: Springer International Publishing, 61-100. doi:10.1007/978-3-03033039-2_3

Conflict of Interest: The authors declare that the research was conducted in the absence of any commercial or financial relationships that could be construed as a potential conflict of interest.

Publisher's Note: All claims expressed in this article are solely those of the authors and do not necessarily represent those of their affiliated organizations, or those of the publisher, the editors and the reviewers. Any product that may be evaluated in this article, or claim that may be made by its manufacturer, is not guaranteed or endorsed by the publisher.

Copyright $(2021$ Ansari, Taha, Dashtipour, Sambo, Abbasi and Imran. This is an open-access article distributed under the terms of the Creative Commons Attribution License (CC BY). The use, distribution or reproduction in other forums is permitted, provided the original author(s) and the copyright owner(s) are credited and that the original publication in this journal is cited, in accordance with accepted academic practice. No use, distribution or reproduction is permitted which does not comply with these terms. 


\section{GLOSSARY}

UAV Unmanned Aerial Vehicles

PAVs Personal Aerial Vehicles

TACS Total Access Communication Systems

LTE Long Term Evolution

GSM Global System for Mobile communications

GPRS General Packet Radio Service

UMTS Universal Mobile Telecommunications Service

KPIs Key Performance Indicators

AV Airborne Vehicle

AWN Airborne Wireless Network

CAVs Connected and Autonomous Vehicles

ITS Intelligent Transport Systems

UE User Equipment

eVTOL electrically-powered Vertical Take-off and Landing

CES Consumer Electronics Show

AI Artificial Intelligence

mVNFs massive Virtual Network Functions

NFV Network Function Virtualization

UTM UAV Traffic Management
LEO Low Earth Orbit

gNBs Base stations

umMTC ultra massive Machine Type Communications

gNB ground NB

aNB aerial-NodeB

AUE Aerial User Equipment

DLT Distributed Ledger Technology

QKD Quantum Key Distribution

ICAO International Civil Aviation Organization

THz Terahertz

V2V Vehicle-to-Vehicle

MIMO Multiple-Input and Multiple-Output

LoS Line-of-Sight

IRS Intelligent Reflecting Surfaces

BVLoS Beyond Visual Line-of-Sight

MEC Multi-access Edge Computing

QoS Quality of Service

NG-RANs Next Generation Radio Access Networks

CAA Civil Aviation Authority

NTN Non-Terrestrial Network. 